\title{
Silicon-on-Insulator Nanowire Resonators for Compact and Ultra-high speed All-optical Wavelength Converters
}

\author{
C. Stamatiadis, K. Vyrsokinos, L. Stampoulidis, I. Lazarou, A. Maziotis, J. Bolten, M. Karl, T. \\ Wahlbrink, P. De Heyn, Z. Sheng, D. Van. Thourhout and H. Avramopoulos
}

\begin{abstract}
We present the development of application specific silicon-on-insulator SOI nanowire ring and racetrack resonators designed to assist semiconductor optical amplifier (SOA)-based all-optical wavelength conversion. We demonstrate the fabrication of fix resonance racetrack resonators exhibiting coupling gaps as low as $160 \mathrm{~nm}$ and tunable ring resonators with coupling gaps $<90 \mathrm{~nm}$. The resonators are cascaded after commercial $40 \mathrm{~Gb} / \mathrm{s}$ SOAs to realize chirp filtering of the optical data signals through high resolution spectral sampling. We experimentally demonstrate inverted and non-inverted SOI resonator-assisted all-optical wavelength conversion at $40 \mathrm{~Gb} / \mathrm{s}$ and $160 \mathrm{~Gb} / \mathrm{s}$ with power penalties $<3 \mathrm{~dB}$.
\end{abstract}

Index Terms - Photonic integration, Micro-ring resonators, Reconfigurable add-drop multiplexers, Semiconductor optical amplifiers, Wavelength converters, Optical signal processing,

\section{INTRODUCTION}

$\mathrm{S}$ ilicon-on-insulator (SOI) has been considered the material of choice for nano-photonic integration [1]. The promising optical properties of SOI together with the complementary metal oxide semiconductor (CMOS) compatible fabrication of optical waveguides have stimulated world-wide research efforts for the implementation of ultra-compact and highdensity photonic integrated circuits [2-6]. In this context, the SOI nanowire waveguide platform has been utilized for the integration of components applicable to wavelength devision multiplexing (WDM) and chip-scale photonic interconnect networks [7]. In these applications high order micro-ring resonators are used to realize add/drop wavelength selective functionalities where high-resolution filtering, band-pass flatness and superior cross-talk are required. Beyond channel add-drop filtering, these passive micro-resonator structures

Manuscript submitted June 1, 2011.

This work has been supported by the European Commission through project ICT-BOOM (FP7-224375) under the $7^{\text {th }}$ Framework Programme.

C. Stamatiadis, K. Vyrsokinos, I. Lazarou, A. Maziotis, and $\mathrm{H}$ Avramopoulos are with the Photonics Communications Research Laboratory, National Technical University of Athens, Zographou, 15773 Athens, Greece (e-mail: cstamat@mail.ntua.gr).

L. Stampoulidis is with Constellex Technology Enablers, Corallia Microelectronics Innovation Center, 12 Sorou Str., Marousi 15125 Athens, Greece

J. Bolten, M. Karl, and T. Wahlbrink are with AMO GmbH, OttoBlumenthal-Straße 25, Aachen, 52074, Germany.

P. De Heyn, Z. Sheng, and D. Van Thourhout are with Photonics Res. Group, INTEC, Ghent Univ-IMEC, 9000, Belgium. have been recently demonstrated to assist switching [8], buffering [9], modulation [10] and all-optical wavelength conversion (AOWC) [11] for next generation photonic routing systems.

In the case of SOA-based micro-resonator assisted wavelength converters, medium index contrast micro-ring resonators with bending sections $\sim 50 \mathrm{um}$ have proven the potential for effective chirp-filtering and WDM-enabled AOWC operation at $40 \mathrm{~Gb} / \mathrm{s}$ [12]. In order to enable even faster bit-rates, higher order coupled resonators are required. These resonators must exhibit micro-scale radius, ultra-sharp spectral profiles and round-trip times comparable to the applied ultra-short pulse widths and the SOI nanowire platform is ideal for meeting these requirements. In [13] we have reported the first experimental evidence on SOA-based $160 \mathrm{~Gb} / \mathrm{s}$ wavelength conversion (WC) assisted by a $3^{\text {rd }}$ order SOI nanowire racetrack resonator with a box-shape spectral profile. The choice of a racetrack was due to the high interaction length of the waveguides (bus-racetrack and racetrack-racetrack) that allows high coupling coefficients with coupling gaps as high as $160 \mathrm{~nm}$ (bus racetrack) and 280 $\mathrm{nm}$ (racetrack-racetrack) respectively.

In this paper, we present the detailed design, fabrication and experimental demonstration of $2^{\text {nd }}$ and $3^{\text {rd }}$ order add/drop SOI micro-resonator structures for all-optical wavelength conversion. All the components were fabricated using high resolution e-beam lithography for patterning 220x450 nm waveguides on $30 \times 30 \mathrm{~mm}^{2}$ SOI chips. Two different component generations are presented; fixed resonance racetrack resonators with 50\% efficient fiber coupling through input-output SU8 tapers and tunable ring resonators with $30 \%$ efficient fiber coupling through input-output grating couplers. Micro-ring resonators were tuned with on-chip integrated titanium heating elements. The integrated heaters were used to alleviate any frequency mismatch of the coupled ring resonators, due to the coupling-induced resonance frequency shifting (CIFS) described in [14]. A number of $>202^{\text {nd }}$ and $3^{\text {rd }}$ order micro-resonator structures as well as $>80$ tapered or grating coupled interfaces have been integrated on a 10.6x 10.4 $\mathrm{mm}^{2}$ area of a $30 \times 30 \mathrm{~mm}^{2}$ SOI chip revealing the high integration density of the approach. We demonstrate $40 \mathrm{~Gb} / \mathrm{s}$ all-optical wavelength conversion using the $2^{\text {nd }}$ order micro structures with power penalties $<2.2 \mathrm{~dB}$. In addition, we demonstrate $160 \mathrm{~Gb} / \mathrm{s}$ all-optical wavelength conversion assisted by the $3^{\text {rd }}$ order micro resonators with power penalties 
$<3 \mathrm{~dB}$. In the future, die-to-wafer bonding and heterogeneous III-V to SOI integration can be employed for large chip-scale integration of the resonator assisted AOWCs [15].

\section{MODELLING}

In this section we describe the design of the micro-resonator structures. The modeling tools that have been employed are: a) ASPIC for generation of ring-resonator transfer functions using waveguide macroscopic parameters, b) VPI for systemlevel AOWC evaluation and c) a beam propagation tool for waveguide size and gap calculation. Firstly physical characteristics, such as ring radius, attenuation of waveguides, group refractive index and power coupling coefficients between nanowires were inserted as parameters into ASPIC. For each parameter combination, ASPIC was generating a corresponding frequency and phase response which was loaded as input to a VPI-based wavelength conversion modeling platform. The VPI setup comprised a SOA with 30 ps recovery time, a filtering module with the ASPIC transfer function and a 2 ps passive delay interferometer (DI). The wavelength conversion process is based on the chirp-filtering method [16]. Figure 1a) depicts the principle operation. A data stream and a local continuous wave (CW) are injected into the SOA for cross gain-phase modulation (XGM-XPM). Due to the finite recovery time of the SOA, the wavelength converted (WC) signal is distorted. To accelerate signal performance, chirp filtering is employed using the response of a higherorder ring resonator. With slight blue-shift offset filtering, an inverted wavelength converted signal is obtained while with larger filter detuning carrier suppression is achieved resulting in a non-inverted waveform. Signal polarity inversion for data rates $>40 \mathrm{~Gb} / \mathrm{s}$ is being performed with a delay interferometer (DI) using its spectral notch for carrier suppression. Far-offset filtering cannot be implemented since filter detuning is larger, losses increase and post-amplification becomes not optimum.

The first simulations were performed at data rates up to 40 $\mathrm{Gb} / \mathrm{s}$. For effective chirp filtering, the drop-transmission ports of $2^{\text {nd }}$ order micro-ring components were utilized with 500 $\mathrm{GHz}$ FSR, $60 \mathrm{GHz} 3 \mathrm{~dB}$ bandwidth and $20 \mathrm{~dB}$ out of band suppression. Figure 1b) depicts the back-to-back data signal entering into the SOA encoded with a pseudo-random bit sequence (PRBS) $2^{7}-1$ and 2ps pulsewidth. When the frequency response of the ring was centered on the peak of the wavelength converted signal, the recovery time of the SOA was evident as illustrated in Figure 1c). With $40 \mathrm{GHz}$ blue shift, the recovery of the converted signal was accelerated leading to an open but inverted eye as shown in Figure 1d).

The cascaded DI with $2 \mathrm{ps}$ differential delay could subsequently restore the polarity to a non inverted format as depicted in Figure 1e). The resulting waveform had very low pedestal and very well shaped pulses. The carrier frequency of the original signal was $193.6 \mathrm{THz}$, while the $\mathrm{CW}$ signal powering the wavelength converting scheme was $193.1 \mathrm{THz}$.

For operation at $160 \mathrm{~Gb} / \mathrm{s}$ the $2^{\text {nd }}$ order ring structure was not suitable due to the narrow $3 \mathrm{~dB}$ bandwidth and low outband suppression filter profile. For this reason a $3^{\text {rd }}$ order ring resonator was designed with $4 \mathrm{THz}$ FSR, $190 \mathrm{GHz}$ bandwidth and $30 \mathrm{~dB}$ out of band suppression. By blue shifting the $3^{\text {rd }}$ order ring resonator $120 \mathrm{GHz}$ off the peak of the converted
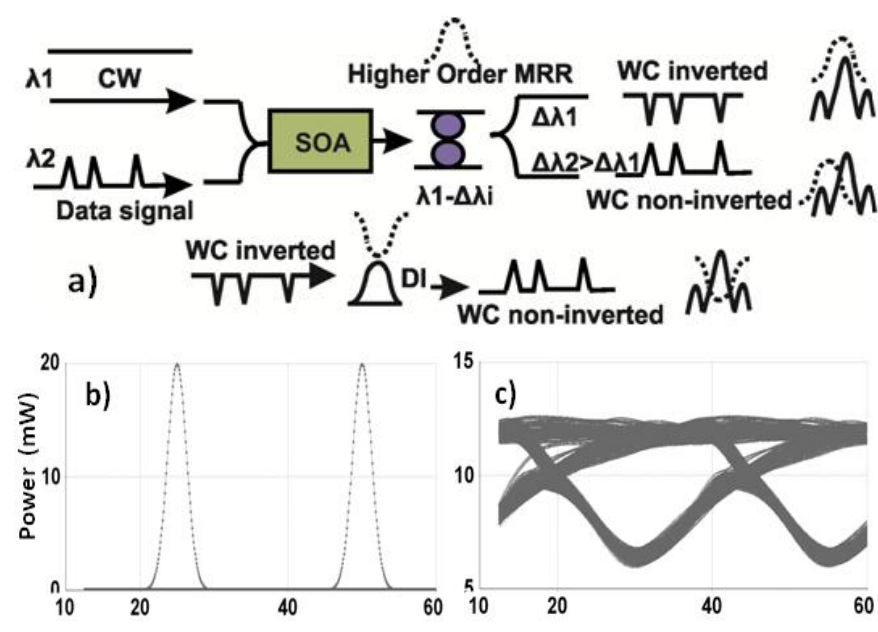

15
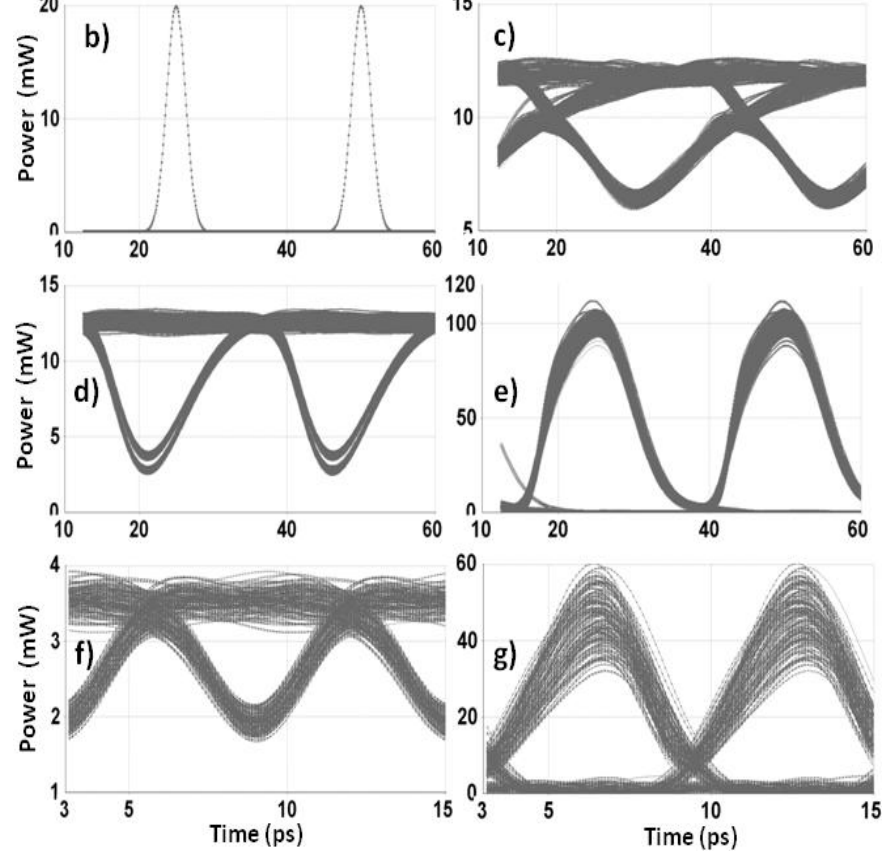

Figure 1: a) Wavelength conversion principle, b) Back-to-back (B2B) signal, c) Recovery of the SOA, d) $40 \mathrm{~Gb} / \mathrm{s}$ inverted WC, e) $40 \mathrm{~Gb} / \mathrm{s}$ Non-Inverted WC, f) $160 \mathrm{~Gb} / \mathrm{s}$ inverted WC, g) $160 \mathrm{~Gb} / \mathrm{s}$ Non-Inverted WC

Table I. Specifications for higher-order micro-resonator structures

\begin{tabular}{lcccc}
\hline Structure & $\begin{array}{c}2^{\text {nd }} \text { Order } \\
\text { Ring }\end{array}$ & $\begin{array}{c}2^{\text {nd }} \text { Order } \\
\text { Racetrack }\end{array}$ & $\begin{array}{c}3^{\text {rd }} \text { Order } \\
\text { Ring }\end{array}$ & $\begin{array}{c}3^{\text {rd }} \text { Order } \\
\text { Racetrack }\end{array}$ \\
\hline $\begin{array}{l}\text { FSR } \\
\text { Bending radius }\end{array}$ & $23 \mu \mathrm{nm}$ & $4 \mathrm{~nm}$ & $27 \mathrm{~nm}$ & $12 \mathrm{~nm}$ \\
$\begin{array}{l}\text { Straight section } \\
\text { length }\end{array}$ & - & $25.5 \mu \mathrm{m}$ & - & $8.5 \mu \mathrm{m}$ \\
$\begin{array}{l}\text { Waveguide } \\
\text { height }\end{array}$ & $220 \mathrm{~nm}$ & $220 \mathrm{~nm}$ & $220 \mathrm{~nm}$ & $220 \mathrm{~nm}$ \\
$\begin{array}{l}\text { Waveguide width } \\
\text { Bus waveguide } \\
\text { width }\end{array}$ & $450 \mathrm{~nm}$ & $450 \mathrm{~nm}$ & $500 \mathrm{~nm}$ & $450 \mathrm{~nm}$ \\
$\begin{array}{l}\text { Gap bus-ring } \\
\text { waveguide }\end{array}$ & $450 \mathrm{~nm}$ & $450 \mathrm{~nm}$ & $300 \mathrm{~nm}$ & $450 \mathrm{~nm}$ \\
$\begin{array}{l}\text { Gap ring-ring } \\
\text { waveguide }\end{array}$ & $195 \mathrm{~nm}$ & $415 \mathrm{~nm}$ & $220 \mathrm{~nm}$ & $280 \mathrm{~nm}$ \\
\hline
\end{tabular}

signal, the effective recovery time was accelerated as illustrated in Figure 1f). Pulse polarity was again restored with a DI obtaining a clear eye diagram with low pedestal as depicted in Figure 1g). The frequencies of the data stream and the CW injected into the AOWC were $194.6 \mathrm{THz}$ and 193.1THz respectively. 


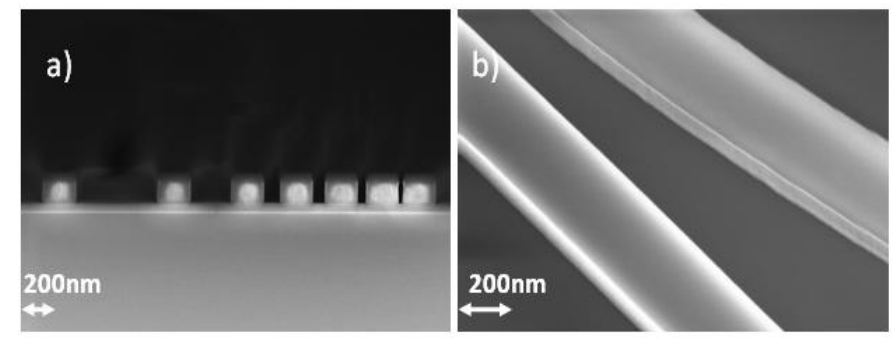

Figure 2: a) Cross-sectional SEM micrograph of etched test structure b) Semicross-sectional SEM micrograph of etched device structures

Given the spectral characteristics of the $2^{\text {nd }}$ and $3^{\text {rd }}$ order micro-rings, the next step was to define the appropriate sizes and waveguide gaps. A beam propagation mode (BPM) custom design tool was employed to extract the specifications listed in Table I for different micro-resonator structures. Racetrack resonators are believed to have more relaxing fabrication tolerances due to their longer interaction length resulting in a larger gap for a certain coupling.

\section{DEVICE FABRICATION ( $1^{\text {ST }}$ RUN)}

The fabrication of high order silicon micro-ring resonator filters requires high resolution lithography. Electron Beam Lithography (EBL) has been employed for fabricating micro structures on SOI substrates with a top silicon layer of $220 \mathrm{~nm}$ on top of a $2 \mu \mathrm{m}$ thick buried oxide. Hydrogen silsesquioxane (HSQ) has been used as negative tone resist to define waveguides, resonators, as well as auxiliary structures such as markers for the alignment of further exposures. A high contrast development process utilizing high concentrated tetramethyl ammonium hydroxide (TMAH) as developer solution has been used [17]. The e-beam dose has been selected to generate correct waveguide width in the HSQ resist. No proximity effect corrections (PEC) were applied. A detailed analysis of the critical dimensions of the design such as coupling gaps, waveguide widths or ring and racetrack widths has been carried out revealing that the resulting changes with respect to the designs critical dimensions were $\pm 5 \mathrm{~nm}$.

The structures have been transferred into the top silicon layer by means of inductively coupled plasma reactive ion etching (ICP-RIE). The etching process used is mainly based on $\mathrm{HBr}$-chemistry. In a first step, pure $\mathrm{HBr}$ is used to achieve a highly anisotropic etch profile. The etching time for this first step is tuned so that a residual top silicon layer of about $10 \mathrm{~nm}$ remains on the substrate. The second step uses an admixture of $\mathrm{O}_{2}$ to increase the selectivity between silicon and silica etching, resulting in a good etch stop on the buried oxide layer [18]. Figure 2a) demonstrates the high quality of the etching process showing a cross sectional SEM micrograph of test structures etched with the same process. Figure $2 \mathrm{~b}$ ) shows a semi-cross-sectional view of an etched sidewall, visualizing the low surface roughness of the structures. Figure $3 a$ ) depicts a $3^{\text {rd }}$ order ring resonator with $3 \mu \mathrm{m}$ bending radius while figure $3 \mathrm{~b}$ ) shows the $75 \mathrm{~nm}$ coupling gap obtained between the bus and ring waveguide. After structure transfer of the resist mask into the substrate material via ICP-RIE, the fabricated chips have again been inspected by SEM. The waveguide width, nominal cross section 450nm x 220nm, has been measured for different types of structures (straight waveguide,

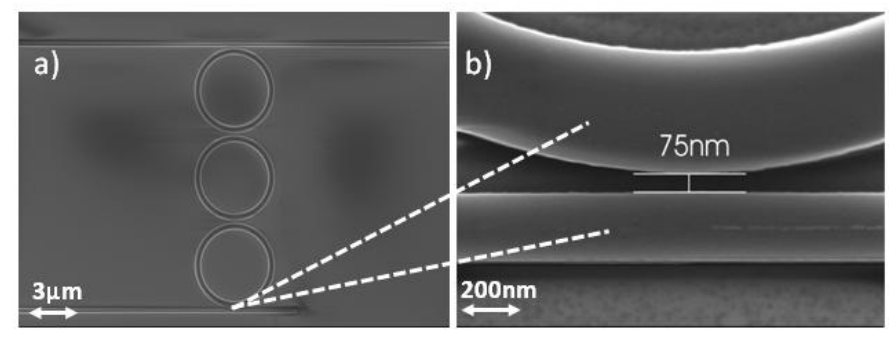

Figure 3: SEM micrograph of a) $3^{\text {nd }}$ Order Ring b) Bus-Ring coupling gap

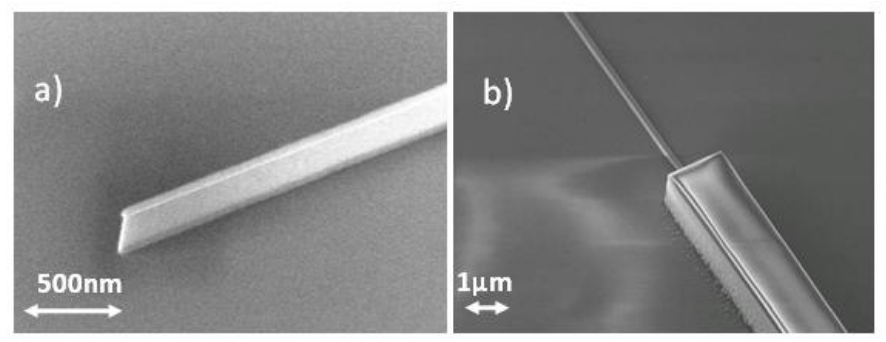

Figure 4: a) Waveguide taper tip structure, b) SU-8-cladded waveguide

ring and racetrack) and in different areas of the chip. A slight increase of the waveguide width measured after the etching compared to the width measured after EBL processing has been observed demonstrating both good critical dimension (CD) control and steepness of the resulting etch profile.

To enable efficient fiber-to-chip coupling, SU8 spot size converters have been integrated into the design [19]. Therefore, the silicon waveguide ends have been designed with narrow tips of about $\sim 40 \mathrm{~nm}$ width. In figure $4 \mathrm{a}$ ) an etched waveguide taper tip is shown in a SEM micrograph. In a second electron beam lithography step, those tapered waveguide ends have been covered with a fiber-size SU-8 cladding waveguide which allows high efficiency coupling, as depicted in Figure 4b). The whole fabrication process, consisting of pattern processing, electron beam exposure, development, pattern transfer and definition of the cladding waveguide has been optimized with respect to different quality parameters such as side wall roughness, critical dimension control and high coupling efficiencies to guarantee a good performance of the devices fabricated.

\section{DEVICE FABRICATION ( $2^{\mathrm{ND}}$ RUN)}

In the next fabrication run, heating elements have been incorporated in the micro-components for a) compensating any fabrication tolerances that would lead to gap variations and frequency mismatch and b) making the wavelength selective switches fully-functional and reconfigurable. The integration of heating elements on top of the SOI photonic layer required however a cladding that would serve as a spacer. The spacer was essential since the metal should not lie directly on top of the SOI photonic layer. The reason is that due to the evanescent field, the light is absorbed by the metal, and thus decreasing the performance of the ring. Figure 5a) shows a cross section of the layer structure: The SOI waveguide (ring) with a cross section of $450 \mathrm{~nm} \times 220 \mathrm{~nm}$ is placed on top of the buried oxide (BOX). An additional $\mathrm{SiO}_{2}$ layer with $800 \mathrm{~nm}$ thickness is deposited by spin coating. The metal that is used for the heating elements is titanium with a thickness of $100 \mathrm{~nm}$. The heating elements are structured by a 


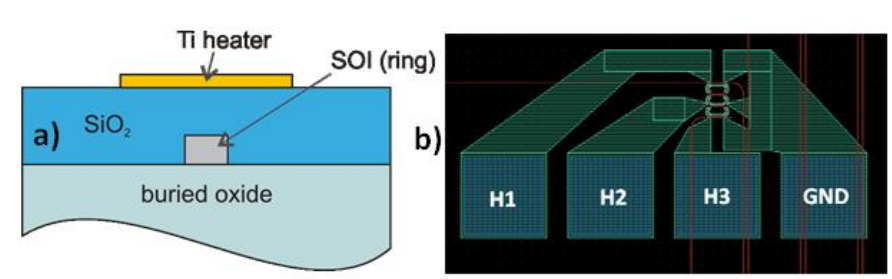

Figure 5: a) Cross section of a SOI waveguide (ring) with $\mathrm{SiO} 2$ cladding and Ti as heating metal on top. b) Layout of heating elements on top of a $3^{\text {rd }}$ order SOI ring resonator
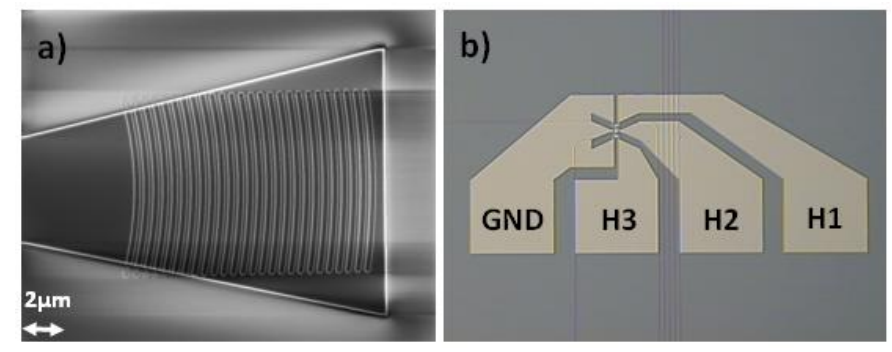

Figure 6: a) SEM picture of fabricated grating coupler, b) Optical microscope image of a $3^{\text {rd }}$ order ring resonator with heating elements

lift-off process and they are designed for effective temperature variation along the ring elements without affecting the coupling regions in a deteriorating way. Their layout for a $3^{\text {rd }}$ order ring resonator is depicted in Figure 5b). All the integrated heaters can be enabled using 4 metal pads: 1 common ground, and 3 pads for driving the heater of each coupled ring resonator.

The fabrication procedure has been started with the definition of waveguide and resonator devices by EBL. To integrate the shallow etched grating couplers, a process flow which allows the combination of fully etched strip waveguides with partially etched grating couplers has been developed. For that purpose, the silicon waveguide ends have been designed as wide triangular area. This area is shown in Figure 6a) and is defined in a first EBL step together with the waveguides and resonators. The defined pattern has been transferred into the top silicon layer by means of inductively coupled plasma reactive ion etching. The top silicon layer has been fully etched down to the buried oxide. Then the grating is realized in the triangular area at the waveguide ends employing additional EBL and ICP-RIE steps. For the definition of the gratings on the pre-structured samples the positive tone electron sensitive resist ZEP 520A has been used. The EBL is followed by shallow etching. An overview of a fabricated grating coupler is shown in Figure 6a). As the overlap of the grating into the buried oxide region is almost equal for both the top and the bottom edge of the coupler, the overlay accuracy between the two different EBL steps is very good. In the next fabrication steps the heater elements were integrated on top of the higher order resonator structures. A spin on glass (SOG) cladding has been chosen for the separation of the waveguide from the heater. The SOG material has been deposited by spin coating. On top of the SOG material the heater elements, depicted in Figure 6b), have again been defined by EBL using PMMA resist. After pattern definition a $100 \mathrm{~nm}$ thick titanium layer has been deposited by electron beam evaporation and the heating elements have been structured by a lift-off process.
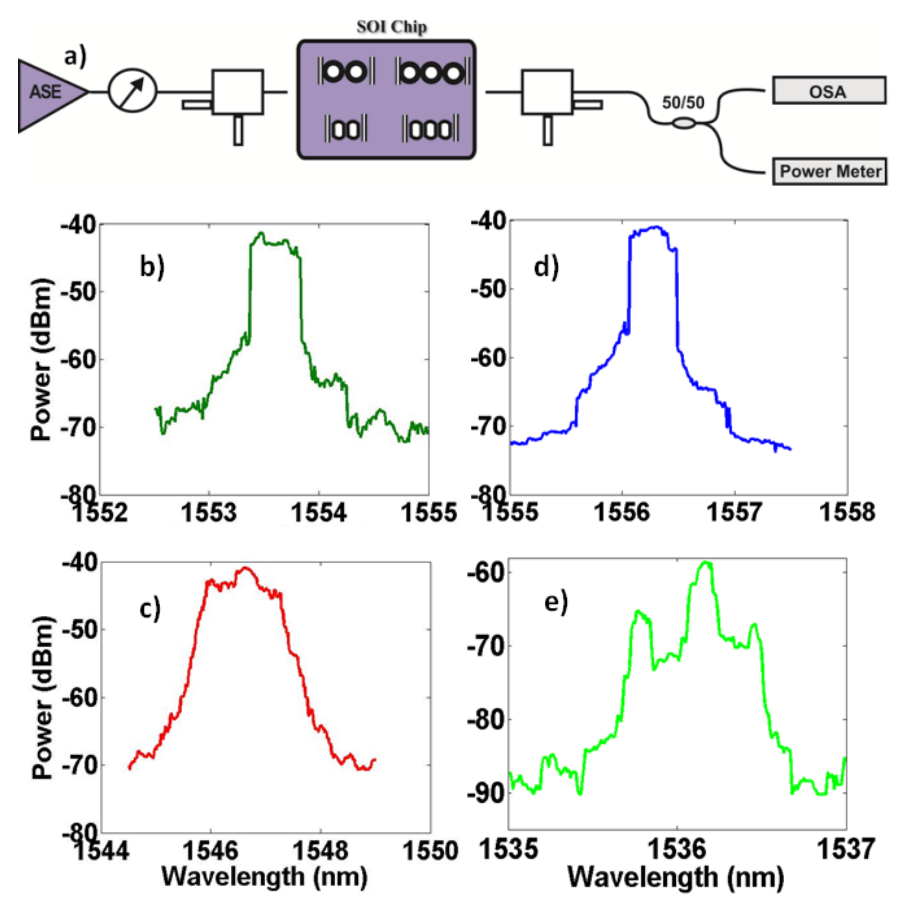

Figure 7: a) Optical characterization setup, Drop transmission responses of b) $2^{\text {nd }}$ Order racetrack, c) $3^{\text {rd }}$ Order racetrack, d) $2^{\text {nd }}$ order Ring, e) $3^{\text {rd }}$ order Ring

Table II. Characterization results for $1^{\text {st }} / 2^{\text {nd }}$ run micro-structures

\begin{tabular}{lcccc}
\hline Structure & $\begin{array}{c}2^{\text {nd }} \text { Order } \\
\text { Parameter }\end{array}$ & $\begin{array}{c}2^{\text {nd }} \text { Order } \\
\text { Racetrack }\end{array}$ & $\begin{array}{c}3^{\text {rd }} \text { Order } \\
\text { Ring }\end{array}$ & $\begin{array}{c}3^{\text {rd }} \text { Order } \\
\text { Racetrack }\end{array}$ \\
\hline FSR & $3.6 / 3.8 \mathrm{~nm}$ & $3.6 / 3.8 \mathrm{~nm}$ & $26 / 28 \mathrm{~nm}$ & $11 / 12 \mathrm{~nm}$ \\
$3 \mathrm{~dB} B \mathrm{~W}$ & $0.4 / 0.6 \mathrm{~nm}$ & $0.5 / 0.7 \mathrm{~nm}$ & $0.3 / 1.4 \mathrm{~nm}$ & $1.4 / 1.4 \mathrm{~nm}$ \\
$\begin{array}{l}\text { Out-band } \\
\text { suppression }\end{array}$ & $13 / 15 \mathrm{~dB}$ & $14 / 16 \mathrm{~dB}$ & $17 / 22 \mathrm{~dB}$ & $25 / 26 \mathrm{~dB}$ \\
$\begin{array}{l}\text { Fiber-to-Fiber } \\
\text { losses }\end{array}$ & $12 / 29 \mathrm{~dB}$ & $11 / 28 \mathrm{~dB}$ & $29 / 40 \mathrm{~dB}$ & $12 / 29 \mathrm{~dB}$ \\
\hline
\end{tabular}

\section{OPTICAL CHARACTERIZATION}

Figure 7(a) depicts the experimental setup for the characterization of the SOI components. Amplified spontaneous emission (ASE) from a $\mathrm{C}$ and L band EDFA was combined for capturing the micro-resonator responses. A single polarization state was preserved utilizing a polarizer with over $30 \mathrm{~dB}$ polarization extinction ratio. The light was coupled into the chip through polarization maintaining lensed fibers and the spectral responses were acquired by an optical spectrum analyzer (OSA) with a resolution of $10 \mathrm{pm}$.

Figure 7 illustrates the drop filter responses from the first generation micro-structures with SU8 cladding. Figures $7 \mathrm{~b}, \mathrm{~d}$ ) depict the spectral profiles of the $2^{\text {nd }}$ order micro-structures with sharp and box-shaped profile and out-band suppression around $14 \mathrm{~dB}$. Figures $7 \mathrm{c}, \mathrm{e}$ ) depict the filtering characteristics of $3^{\text {rd }}$ order resonators. The $3^{\text {rd }}$ order racetracks exhibit a wellshaped resonance with optimum spectral overlap between the individual coupled racetracks and a higher out-band suppression with respect to $2^{\text {nd }}$ order elements. The $3^{\text {rd }}$ order micro-ring resonators on the other hand reveal a frequency mismatch - with degenerated peaks- clearly visible in Figure 


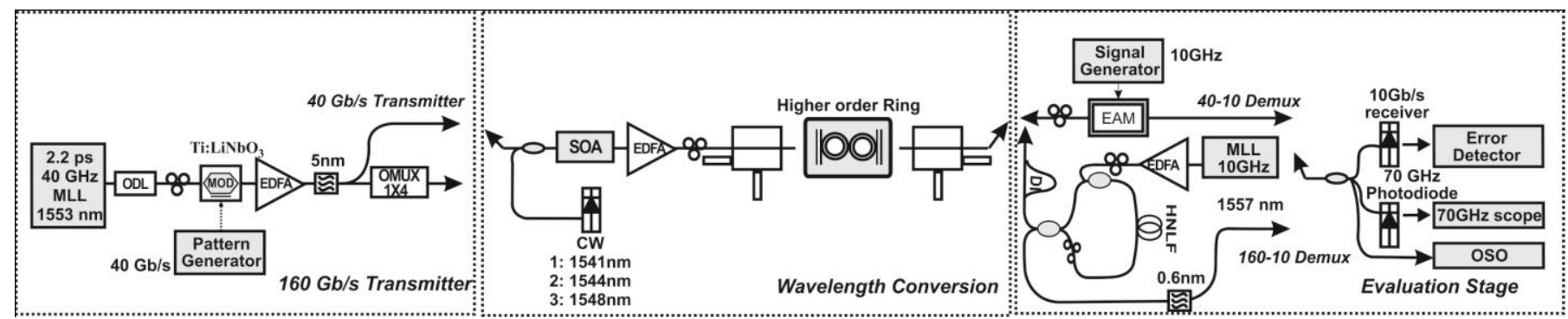

Figure 9: Experimental setup for evaluation of $2^{\text {nd }}$ and $3^{\text {rd }}$ order micro-resonator components

7e). This frequency mismatch can be attributed to the coupling-induced resonance frequency shifting, counteracted by design [14], and to lithographic imperfections. The lower insertion loss measured in racetrack resonators compared to that in ring resonators was attributed to the longer coupling regions between the bus-racetrack and racetrack-racetrack waveguides, the larger spacing between the bus-racetrack and racetrack-racetrack waveguides as well as to the their more relaxing fabrication tolerances. Table II outlines some additional values collected during optical characterization. The free-spectral range measured was near the design specifications of Table I, while the 3-dB bandwidth and outband suppression was sufficient for $40 \mathrm{~Gb} / \mathrm{s}$ and $160 \mathrm{~Gb} / \mathrm{s}$ system tests. The fiber-to-fiber losses have been calculated as low as $\sim 11 \mathrm{~dB}$ verifying the $50 \%$ SU8 coupling efficiency. The high-loss obtained for the $3^{\text {rd }}$ order ring resonator was attributed to the ring resonator spectral mismatch.
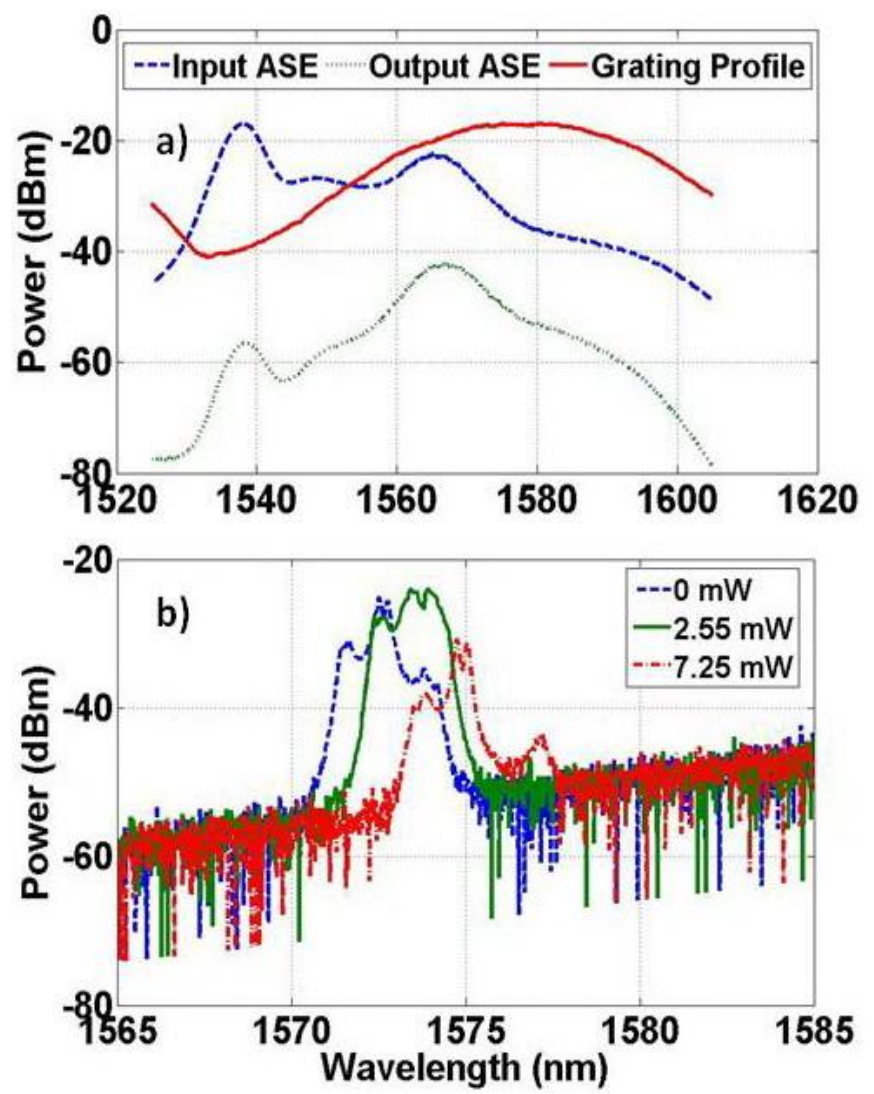

Figure 8: a) Grating coupler filter response, b) Wavelength tuning of $3^{\text {rd }}$ order ring resonator when heater $\mathrm{H} 1$ is enabled
This frequency mismatch observed was corrected with the integration of Ti heaters on top of the ring structures. For their fabrication and metal deposition, an additional $\mathrm{SiO}_{2}$ layer was required precluding the use of SU8 tapers for input-output coupling. As an alternative, grating couplers were fabricated with 30\% efficiency and spectral profile depicted in Figure $8 a)$. It is clearly shown that the response was optimized for wavelengths centred to $1580 \mathrm{~nm}$. Figure 8b) illustrates the wavelength tuning characterization results for the $3^{\text {rd }}$ order ring resonator. Without applying voltage in the integrated heating element, three major peaks were evident due to the spectral mismatch of the ring structures. When increasing the current at the heater H1 (Figure 5b), the left resonance peak of the underlying SOI ring was red-shifted $2 \mathrm{~nm}$ and the center peak $1 \mathrm{~nm}$ resulting to frequency overlapping with the third ring resonance. The reason for the spectral shift of two resonances with a single heater is attributed to the lack of thermal isolation between the coupled ring resonators as well as to their very close proximity $(<90 \mathrm{~nm})$. Assuming a maximum power of approx. $7.25 \mathrm{~mW}$ a tuning range of 5.6 $\mathrm{nm}$ was obtained. Table II outlines also the optical characterization results for the $2^{\text {nd }}$ run devices. Similar spectral characteristics have been observed but with rather higher losses due to the utilization of grating couplers. The typical fiber-to-fiber loss for a reference waveguide was $\sim 17 \mathrm{~dB}$. The higher $3 \mathrm{~dB}$ bandwidth and the out-band suppression value for the $3^{\text {rd }}$ order ring resonator was attributed to properly tuned and coupled resonances using the integrated heating elements.

\section{EXPERIMENT}

\section{A. $40 \mathrm{~Gb} / \mathrm{s}$ system tests using $2^{\text {nd }}$ order SOI resonators}

Figure 9 shows the experimental setup used for the system evaluation of the micro-resonators. The $40 \mathrm{~Gb} / \mathrm{s}$ data signal was generated by a $2.2 \mathrm{ps}, 40 \mathrm{GHz}$, tunable, mode-locked laser modulated with a $2^{7}-1$ pseudo-random bit sequence. A pigtailed commercially available SOA with a gain recovery time of $30 \mathrm{ps}$ was used for cross-gain and -phase modulation. A tunable laser provided the continuous wave (CW) signals. Fiber-to-chip coupling was performed through standard single-mode lensed fibers. The data signal was injected into the SOA together with the $\mathrm{CW}$ light using an optical combiner. Due to the finite recovery time of the SOA, the wavelength converted signal was distorted causing inter symbol interference (ISI). By detuning the $\mathrm{CW}$ to a larger wavelength away from the micro-resonator drop resonance the effective recovery time was accelerated suppressing inter- 

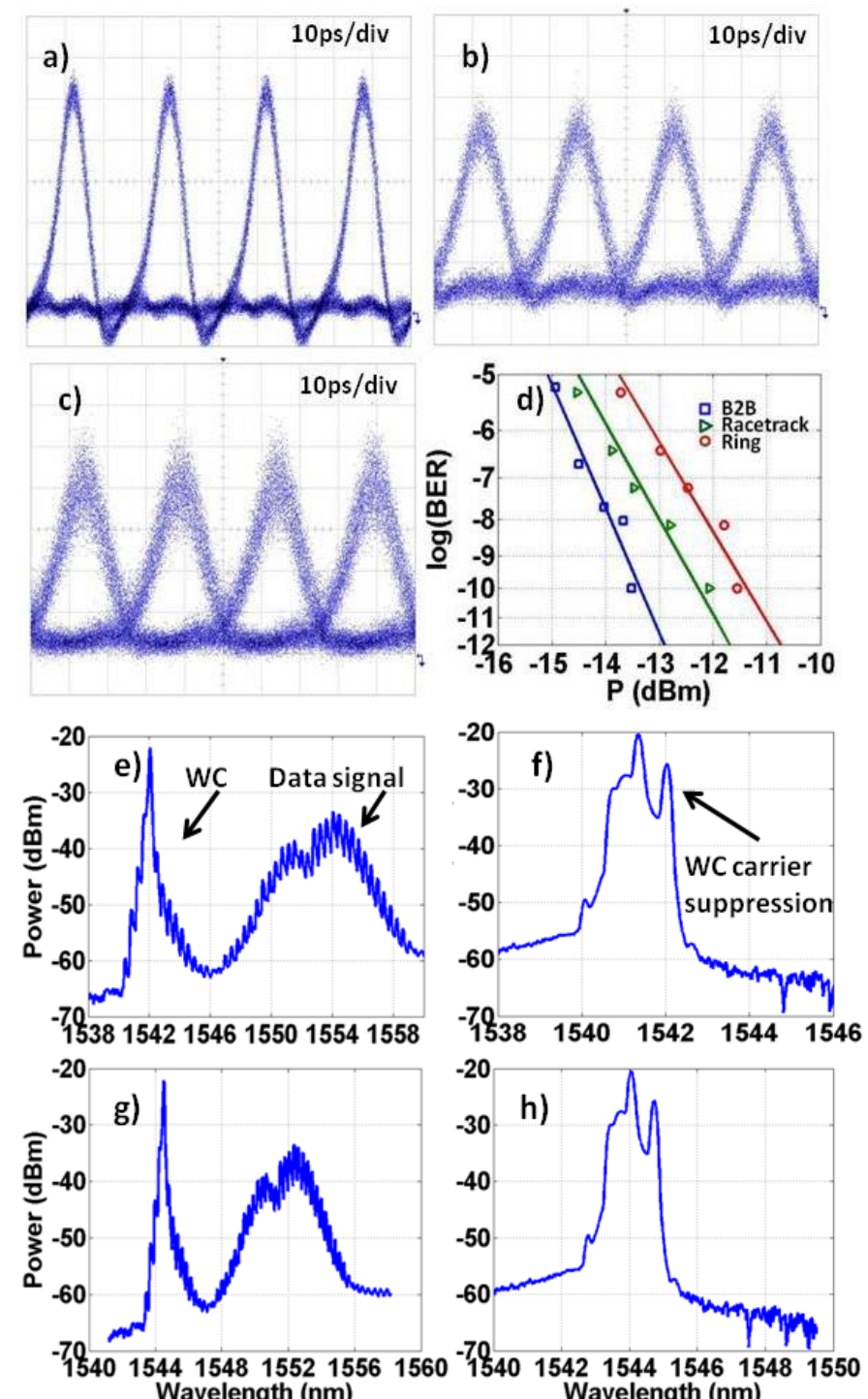

Wavelength $(\mathrm{nm})$

Figure 10: a) 40Gb/s B2B, b) WC with $2^{\text {nd }}$ order racetrack, c) WC signal with $2^{\text {rd }}$ order ring, d) BER curves, Spectra e-f) after SOA and $2^{\text {nd }}$ order racetrack, g-h) after SOA and $2^{\text {nd }}$ order ring respectively

symbol interference. The wavelength converted signal however was in inverted mode since the $\mathrm{CW}$ carrier was dominant with respect to the data harmonic. Signal polarity restore was implemented by further $\mathrm{CW}$ detuning so as the falling edge of the micro-resonance to coincide with the $\mathrm{CW}$ carrier. This had as a consequence the carrier component to be suppressed and the data harmonic to become dominant [20]. After polarity inversion the wavelength converted signal was evaluated with bit-error-rate (BER) measurements after performing demultiplexing to $10 \mathrm{~Gb} / \mathrm{s}$ using an electroabsorption modulator (EAM).

Figure 10a) illustrates the eye diagram of the $40 \mathrm{~Gb} / \mathrm{s}$ backto-back data signal. By detuning the $\mathrm{CW} 0.7 \mathrm{~nm}$ off the $2^{\text {nd }}$ order racetrack filter response, the wavelength converted signal of Figure 10b) was recorded. The waveform had additional noise since inversion was implemented with farfiltering resulting to low output power and not optimum amplification. Figure 10c) shows the eye diagram obtained using the $2^{\text {nd }}$ order micro-ring resonator. The performance deterioration is due to the higher insertion loss and smaller
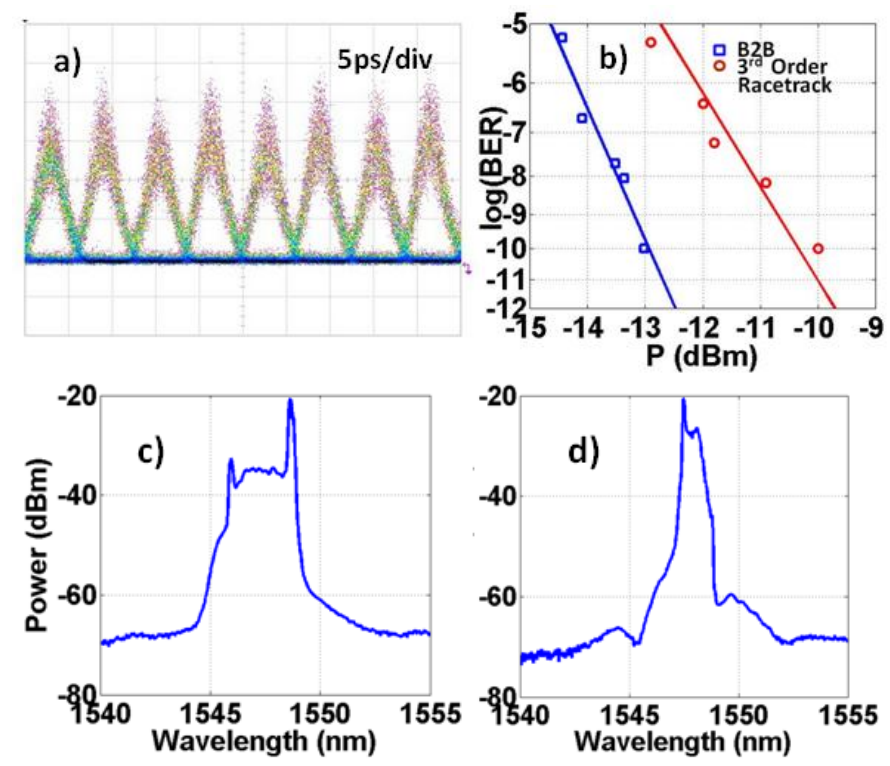

Figure 11: a) $160 \mathrm{~Gb} / \mathrm{s}$ wavelength converted signal using a $3^{\text {rd }}$ order racetrack, b) BER curves for B2B and WC signal, Spectra c) Inverted WC signal, d) Non-Inverted signal after the DI

out-band suppression (Table II) of the ring resonator. The broadening of the pulses for both resonant configurations was attributed to the relative narrow $3 \mathrm{~dB}$ bandwidth $\sim 0.4 \mathrm{~nm}$. Figure 10d) depicts the BER curves. Error-free operation was achieved for all the channels with $1.1 \mathrm{~dB}$ and $2.2 \mathrm{~dB}$ power penalty for the racetrack and ring resonator respectively. Figure 10e) shows the spectra of the $40 \mathrm{~Gb} / \mathrm{s}$ wavelength converted and data signal after the SOA. In order to perform chirp filtering as well as polarity inversion the response of a $2^{\text {nd }}$ order racetrack was employed, blue-shifted with respect to the WC carrier. Since the falling edge of the $2^{\text {nd }}$ order racetrack had a sharp spectral profile, the blue-shift filtering had as a consequence the WC carrier suppression and the polarity inversion of the wavelength converted signal in timedomain. Figure 10f) depicts the $40 \mathrm{GHz}$ harmonic which has become dominant and the WC carrier that has been effectively suppressed. Figures $10 \mathrm{~g}-\mathrm{h}$ ) illustrate the wavelength conversion spectra using a $2^{\text {nd }}$ order ring resonator. Initial and converted wavelengths for both cases were (1) 1553 to $1541 \mathrm{~nm}$ and (2) 1553 to $1544 \mathrm{~nm}$.

\section{B. $160 \mathrm{~Gb} / \mathrm{s}$ system tests using $3^{\text {rd }}$ order devices}

Figure 9 illustrates the experimental setup used for the 160 $\mathrm{Gb} / \mathrm{s}$ system measurements. The $160 \mathrm{~Gb} / \mathrm{s}$ data signal was generated by time multiplexing the $40 \mathrm{~Gb} / \mathrm{s}$ transmitter through a fiber-interleaver. Using this stream together with a local CW, wavelength conversion was performed in the SOA. The slow recovery time was accelerated by employing the filter response of the $3^{\text {rd }}$ order racetrack as chirp-filtering element. Signal polarity was subsequently restored utilizing a 2 ps delay interferometer. At the receiver side the wavelength converted signal was evaluated after performing 160 to $10 \mathrm{~Gb} / \mathrm{s}$ optical demultiplexing using a non-linear loop mirror (NOLM). The non-linear element of the NOLM was $100 \mathrm{~m}$ of HNLF with $10 \mathrm{~W}^{-1} \mathrm{~km}^{-1}$ non linear parameter and 1.21 $\mathrm{ps} / \mathrm{nm} / \mathrm{km}$ dispersion. As control signal into the NOLM, a 
mode-locked laser at $1557 \mathrm{~nm}$ was utilized emitting $1.3 \mathrm{ps}$ optical pulses at $10 \mathrm{GHz}$ repetition rate. Figure 11a) shows the non-inverted wavelength converted signal recorded with an Optical Sampling Oscilloscope (OSO). From the OSO trace, it is evident that the out-band suppression and bandwidth of the $3^{\text {rd }}$ order racetrack was sufficient for speeding up the SOA and enable $160 \mathrm{~Gb} / \mathrm{s}$ operation. Figure 11b) depicts the BER curves obtained after optical demultiplexing. The power penalty was measured $\sim 3 \mathrm{~dB}$, which is close to the power penalty obtained using bulk, and customized optical band-pass filters for chirp filtering [16]. Figures 11c-d) illustrate the spectra of the $160 \mathrm{~Gb} / \mathrm{s}$ wavelength converted signal in the inverted and non-inverted mode respectively. In fact figure 11c) presents the spectrum after the $3^{\text {rd }}$ order racetrack when $1.2 \mathrm{~nm}$ blue-shift offset filtering has been performed. In order to restore the polarity of the wavelength converted signal in time domain a delay interferometer was applied whose spectral notch was centered to the WC carrier. Figure 11d) depicts the effective carrier suppression by the delay interferometer. Signal polarity inversion using only the spectral response of the $3^{\text {rd }}$ order racetrack was not feasible since the required offset filtering was too large resulting to high losses and not-optimum amplification. The $3^{\text {rd }}$ order ring structures could not be experimentally tested due to the high fiber-to-fiber losses $(\sim 39 \mathrm{~dB})$. Our current work is focused on the grating coupler design and fabrication optimization [21] in order to minimize loss and allow subsequent system testing.

\section{CONCLUSION}

We have demonstrated the design, fabrication and experimental evaluation of nano-photonic structures for micro-resonator assisted all-optical wavelength converters. Firstly, we have specified all the physical parameters using appropriate modeling tools. We reported two fabrication runs based on high resolution e-beam lithography for the development of $2^{\text {nd }}$ and $3^{\text {rd }}$ order micro-ring and racetrack resonators. We demonstrate error-free wavelength conversion up to $160 \mathrm{~Gb} / \mathrm{s}$ employing the nano-wire periodic filters. Power penalties $<3 \mathrm{~dB}$ have been obtained making the use of micro-resonators promising for scalable and power efficient AOWC.

\section{ACKNOWLEDGEMENTS}

This work was supported by the European Commission through ICT-BOOM project under the $7^{\text {th }}$ Framework Programme. The authors gratefully acknowledge Alnair Labs for providing supporting equipment.

\section{REFERENCES}

[1] B. Jalali, S. Fathpour, "Silicon Photonics", IEEE J. Lightwave Technology, 26, 4600-4615, (2006).

[2] N. Izhaky, M.T Morse, S. Koehl, O. Cohen, D. Rubin, A. Barkai, G. Sarid, R. Cohen and M.J. Paniccia, "Development of CMOS-Compatible Integrated Silicon Photonics Devices", IEEE J. Sel. Top. Quantum Electron., 12, 1688-1698, (2006).

[3] S. Janz, P. Cheben, D. Dalacu, A. Delage, A. Densmore, B. Lamontagne, M.J Picard, E. Post, J.H Schmid, P. Waldron, D.X. Xu, K.P. Yap and W.N Ye, "Microphotonics Elements for Integration on the Silicon-on-Insulator Waveguide Platform", IEEE J. Sel. Top. Quantum Electron., 12, 1402-1415, (2006).

[4] T. Tsuchizawa, K. Yamada, H. Fukuda, T. Watanabe, J. Takahashi, M. Takashi, T. Shoji, E. Tamechica, S. Itabashi and H. Morita,
"Microphotonics Devices Based on Silicon Microfabrication Technology", IEEE J. Sel. Top. Quantum Electron., 11, 232-240, (2005).

[5] W. Bogaerts, P. Dumon, D. Van Thourhout, D. Taillaert, P. Jaenen, J. Wouters, S. Beckx, V. Wiaux and R.G Baets, "Compact WavelengthSelective Functions in Silicon-on-Insulator Photonic Wires", IEEE J. Sel. Top. Quantum Electron., 12, 1394-1401, (2006).

[6] U. Plachetka, N. Koo, T. Wahlbrink, J. Bolten, M. Waldow, T. Plötzing,, M. Först, and H. Kurz, "Fabrication of Photonic Ring Resonator Device in Silicon Waveguide Technology Using Soft UV-Nanoimprint Lithography", IEEE Photon. Technology Letters., 20, 490-492, (2008).

[7] S.T. Chu, B.E Little, V.Van, J.V Hryniewicz, P.P Absil, F.G Johnson, D. Gill, O. King, F. Seiferth, M. Trakalo and J. Shanton, "Compact Full Cband tunable filters for $50 \mathrm{GHz}$ channel spacing based on high-order micro-ring resonators", in Proc. OFC 2004, Los Angeles, (CA), USA, PDP9.

[8] B.G. Lee et. al, "High-Speed 2x2 Switch for Multiwavelength SiliconPhotonic Networks-On-Chip," J. Lightwave Technology, 27, 29002907, (2009).

[9] F. Xia, L. Sekaric, Y. Vlasov, "Ultra-compact optical buffers on a silicon chip," Nature Photonics, 1, 65-71 (2007).

[10] N. Ophir, K. Padmaraju, A. Biberman, L. Chen, K. Preston, M. Lipson and K. Bergman, "First Demonstration of Error-Free Operation of a Full Silicon On-Chip Photonic Link," in Proc. OFC 2011, Los Angeles (CA), USA, OWZ3.

[11] K. Vyrsokinos, L. Stampoulidis, Z. Sheng, E. Kehayas, P. Bakopoulos, D. Petrantonakis, C. Stamatiadis, Ch. Kouloumentas, P. Zakynthinos, R. Dekker, E. J. Klein, D. Van Thourhout, M.T. Kothorst and H. Avramopoulos, "Higher-order micro-ring resonator assisted wavelength converters for scalable and power efficient photonic routers", in Proc. ECOC 2009, Vienna, Austria, P2.08.

[12] C. Stamatiadis, D. Petrantonakis, P. Bakopoulos, E. Kehayas, P. Zakynthinos, C. Kouloumentas, L. Stampoulidis, Z. Sheng, E. Kehayas, P. Bakopoulos, D. Petrantonakis, C. Stamatiadis, Ch. Kouloumentas, P. Zakynthinos, R. Dekker, E. J. Klein, D. Van Thourhout, M.T. Kothorst and H. Avramopoulos, "First Demonstration of WDM-enabled AllOptical Wavelength Conversion with a SOA and a 2nd Order Micro-ring Resonator ROADM," in Proc. OFC 2009, San Diego (CA), USA, PDPA8.

[13] C. Stamatiadis, K. Vyrsokinos, L. Stampoulidis, A. Maziotis, Z. Sheng, D. Van Thourhout, J. Bolten, M. Karl, T. Wahlbrink and H. Avramopoulos, "All-Optical Wavelength Conversion at $160 \mathrm{~Gb} / \mathrm{s}$ Using an SOA and a $3^{\text {rd }}$ Order SOI Nanowire Periodic Filter", in Proc. IEEE Photonics Society Annual Meeting 2010, Denver, USA.

[14] M. Popovic, M. Watts, T. Barwicz, P. Rakich, L. Socci, E. Ippen, F. Kartner and H. Smith, " High-Index-Contrast, Wide-FSR MicroringResonator Filter Design and Realization with Frequency-Shift Compensation", in Proc. OFC 2005, Anaheim, (CA), USA, OFK1.

[15] L. Liu, T. Spuesens, D. Van Thourhout, G. Morthier, L. Grenouillet, N. Olivier, J. Fedeli, P. Romeo, P. Régreny, F. Mandorlo and R. Orobtchouk, "200mm wafer scale III-V/SOI technology for all-optical network-on-chip and signal processing", 7th IEEE International Conference on Group IV Photonics (GFP), Beijing (2010).

[16] Y. Liu, E. Tangdiongga, Z. Li, H. de Waardt, A.M.J. Koonen, G.D. Khoe, H.J.S. Dorren, "Error-free $320 \mathrm{Gbit} / \mathrm{s}$ SOA-based wavelength conversion using optical filtering," in Proc. OFC 2006, Anaheim (CA), USA, PDP28.

[17] W. Henschel, Y.M. Georgiev, H. Kurz, "Study of a high contrast process for hydrogen silsesquioxane as a negative tone electron beam resist", J. Vac. Sci. Technol. B21, 5, 2018-2024, (2003).

[18] T. Wahlbrink, T. Mollenhauer, Y.M. Georgiev, W. Henschel, J.K. Efavi, H.D.B. Gottlob, M.C. Lemme, H. Kurz, J. Niehusmann, P. Haring Bolivar, "Highly selective etch process for silicon-on-insulator nano devices", Microelectronic Engineering, 78-79, 212-217, (2005).

[19] T. Wahlbrink, W.S. Tsai, M. Waldow, M. Först, J. Bolten, T Mollenhauer and H. Kurz, "Fabrication of high efficiency SOI taper structures", Microelectronic Engineering ,86, 1117-1119, (2009).

[20] J. Dong, X. Zhang, S. Fu, J. Xu, P. Shum and D. Huang, "Ultrafast AllOptical Signal Processing Based on Single Semiconductor Optical Amplifier and Optical Filtering, IEEE J. Sel. Top. Quantum Electron., 14, 770-778, (2008).

[21] D. Vermeulen, S. Selvaraja, P. Verheyen, G. Lepage, W. Bogaerts, P. Absil, D. Van Thourhout, G. Roelkens, "High-efficiency fiber-to-chip grating couplers realized using an advanced CMOS-compatible siliconon-insulator platform",Optics Express, 18, p.18278-18283, (2010) 